\title{
Problem of Relation Between Brain and Mind in Physiology, Medicine and Psychology
}

\section{Reshetnikov MM*}

The East-European Psychoanalytic Institute, St. Petersburg, Russia

"Corresponding Author: Reshetnikov MM, The East-European Psychoanalytic Institute, St. Petersburg, Russia, Tel: + 781223528 57; E-mail: veip@yandex.ru

Received: 29 September 2017; Accepted: 13 October 2017; Published: 23 October 2017

\begin{abstract}
In this paper, traditional concepts of relation between mental activity and brain activity are reviewed, and hypothesis of brain as a biological interface is developed, which was suggested by the author earlier (2008). Approaches to research of human mind in areas of physiology, psychophysiology, psychology and psychiatry are analysed, as well as their applications to therapy of mental disorders. The author suggests two main models of mental disorder based on clearer distinction between organic (brain) pathology and mental disorder resulting from informational nonmaterial influence on psyche rather than brain. Difference between nervous and psychic functioning of organism and personality is outlined, and mental activity is viewed as an acquired function programmed by social informational environment. The question of aim of psychopharmacological influence is raised.
\end{abstract}

Keywords: Biological interface; Information; Brain; Nerves; Psyche; Psychosomatics; Psychopharmacology; Symbolization; Software and hardware; Brain structures; Psychic structures

\section{Relation and Interconnection Between Brain and Mind}

Relation and interconnection between brain and mind has been one of the most mysterious and still unresolved problems for last two thousand years. Prior to Hippocrates, is had been generally accepted that mental phenomena are closely connected to brain functioning, but then Hippocrates modified this idea, and in the next centuries it was assumed that brain is a location of all mental processes. It will be argued that difference between the two formulations, which often goes unnoticed, is crucial. Despite significant progress of research in this area, 
Hippocrates's idea has been dominating physiology, psychiatry and psychology for last two thousand years. The only exception could be found in some modalities of psychotherapy that view psyche as an epiphenomenon.

Before discussing the idea of epiphenomenon, let us outline the main stages of development of contemporary scientific concepts. On the basis of I.M. Sechenov and I.P.Pavlov's theories, two new areas on the edge of psychology and physiology were formed, that is, physiology of higher nervous activity and psychophysiology. It should be mentioned that representatives of these two approaches spent almost a century trying to explain mental activity exclusively by excitation and inhibition, reflexes and biochemical reactions, but now they, with the exception of very few authors, have restricted their area of interest to studying brain structures as a basis of mental activity. Let us stress it: these structures are only basis for mental activity.

However, psychiatry and psychopharmacology still base their approach to psychopathology on traditional theory of higher nervous activity, which still identifies mind with brain and focuses on anatomical structure of nervous system, location of functions in brain cortex, transmitters, biochemical reactions and neuromediators. This identification is a basis for all traditional methods of therapy of mental disorders, which in most mental hospitals means chemical (psychopharmacological) influence on brain and on synaptic transmission.

Let me remind you that in 2008 I suggested that human brain can be viewed as a biological interface [1, 2]. The hypothesis draws a parallel between brain and computer hardware, while mind can be compared to software, and process of education can be considered a kind of programming. Mental activity is viewed as a version of informational exchange and interaction, and physical and physiological symptoms as embodied representations of soul life, which do not give any specific information about individual's thoughts, ideas and experiences and psychic contents in general.

From this perspective, previous widely accepted attempts to study mental activity by means of EEG can be compared to measuring voltage and resistance of a TV-set in attempts to get information about TV programs. Approaches to mental processes (memory, attention and thinking) by means of psychological tests seem to be doubtful as well. What is measured and quantified as mental processes is external work of intellect, a result of its ability to process information (parallel to computer's hardware, such as operative memory capacity and speed of work). Drawing conclusions about mental processes from such results can be compared with attempts to understand biochemical and physiological processes in bodily tissues by observing individual's external physical activity, such as digging a trench. Mental activity could be understood only by means of self-observation and reflection, and these methods for long period of time were viewed as idealistic, subjective and non-scientific by psychology; however, this attitude to self-knowledge methods has changed in recent years.

It should be also mentioned that contemporary science considers information a non-material factor, while data carriers (biological, paper, electronic etc.) are material. However, non-material information has a number of quantitative and qualitative characteristics. It can be neutral, emotionally charged, scaring, truthful, false etc., but all these characteristics are revealed only when there is a subject perceiving this information. There is no such thing as 
an information carrier without a perceiving subject. Only alive beings, especially human beings, can be perceiving subjects of information, its creators, its carriers and its verifiers.

Wide-spread concept of brain as location of all psychic functions has given birth to a number of false assumptions which permeate everyday speech; in science, it has led to well-known phenomenon of "theory overload", when data are rejected when they contradict a dominating theory. There are some well-known phrases like "something is wrong with my nerves", although nerves are just mediators; or "it has come into my head" although actually it has come to my mind etc. In general, both in science and in everyday life nervous and psychic phenomena are often perceived as indiscriminate.

The author has managed to find crucial differences between nervous system and mind. There is a number of differences, and the main one is the following: mind is able to distinguish imaginary stimuli from real ones. Nervous system reacts almost in the same way to both kinds of stimuli. It is the basis of suggestion and auto-suggestion techniques: for instance, when individual imagines putting his hand into hot water, it will lead to increased temperature in his hand, and imagining running a hundred-meter race will make his pulse rise. However, very few specialists, except for psychotherapists, know that psychosomatic pathology develops in the same way and follows individual scheme of symbolization (inner psychic programming). What was "taken to heart" can result in cardiac pathology; a "swallowed up" offense can be a factor in bulimia or anorexia, breathing difficulty as a reaction to shocking news might lead to asthmatic attacks, and "weakness in knees" might result in joints pain. In general, individual system of psychic trauma symbolization is akin to repetitive suggestion, or rather auto-suggestion. It is possible to agree or disagree with it, but I suppose that even people unfamiliar with the area of psychotherapy might have observed how an unbearable psychological burden would make somebody’s back hunched.

Let us turn to psychopathology again. We seem to be still unable to clearly differentiate its two distinct types, that is:

1. Psychopathology as a result of organic damage of brain due to infectious disease, sclerotic processes, oncological disease etc. Thus, brain as information carrier (parallel to computer hardware) gets damaged. In such a case, even external behavioural or psychic manifestations could help to easily determine location of a damaged area of brain, and approaches of biological medicine are adequate: brain needs to be treated by means of chemical substances, surgery, radiation or lasers etc.

2. Psychopathology as a result of informational damage to psyche itself, that is, a non-material factor (such as individually significant psychic trauma) damages non-material and previously normally functioning mind, just as a computer virus damages previously functional software. The most vivid example is a reported "poisoning" of schoolchildren by neuro-paralyzing gas in a few schools in Chechnya in SeptemberDecember 2005, which appeared to be a typical case of mass psychic contagion by false ideas. Ethnic conflicts follow the same paranoid scenario [3].

In contrast to the first group, mental disorders depend on individual specifics of a subject and can widely vary from slight dysphoria to severe autism or persistent paranoia. Therapy should consist of informational influence on damaged psychic structures rather than brain structures. Chemical influence aimed at "hardware" will bring no result in this case, for psychic content would stay unchanged. 
There is a number of detailed and well-based classifications of psycho-pharmacological medicines, but they can be also divided into two groups: the first aims at numbing mental experience (in parallel to reducing the brightness of the computer screen on which information is projected); and the second stimulates mental activity and thus increases brightness of "the screen". But they do not change content of mental sphere (that is, information).

It would be wrong to keep a negative attitude towards psychopharmacology or to deny its success. The author is not against this approach but objects its ungrounded (up to $60 \%$ of such prescriptions are made by GPs), long-term, isolated and uncontrolled use. Isolated and uncontrolled use means that it is applied without systematic control from psychiatrist in collaboration with psychotherapist who would pay attention to psychopharmacological effects influencing the patient's condition, including side effects. When the individual suffers physically or psychologically, first of all a kind of anaesthesia is required. However, in case of psychic suffering the main task is psychic influence by psychiatrist and therapist on condition and contents of the patient's mental sphere.

Let us give one more argument to support the abovementioned hypothesis. Studies of feral children (also known as "Mowgli") shows that normal human mind, as well as bipedalism, cannot develop without early interaction with social environment or without linguistic programming of the child's brain by his environment. It allows us to make one more conclusion: healthy brain is a necessary but not sufficient condition of development and adequate functioning of human mind. Feral children have functional instincts and reflexes, which are genetically determined in contrast to conscious activity that is an acquired function developing only in social informational environment. Let us add that feral children internalize behavioural programs and language (system of informational exchange) of animal group in which they live, thus confirming the ideas which the author develops here.

When these ideas were expressed for the first time, one of my colleagues described them as anti-psychiatric. This is not true. The author has sincere respect to psychiatrists working with the most difficult patients. It does not prevent me, however, from critically reviewing established views, theories and methods of therapy. The author by no means claims possession of the final truth but encourages his colleagues to participate in critical discussion of the problem and the suggested hypothesis.

\section{References}

1. Reshetnikov MM. Mental Disorder. - St-Petersburg: the EEPI (2008): pp 272.

2. Reshetnikov MM. Critical Post-Materialism in Psychology and Psychiatry. Neurological Bulletin 43 (2011): 66-69.

3. Volkan V. Traumatized Societies. In: Violence and Dialogue? Psychoanalytic Insight on Terror and Terrorism. - London, International Psychoanalytic Association (2003): 217-237. 